\title{
Stimulated Emission Pumping Enabling Sub-Diffraction-Limited Spatial Resolution in CARS Microscopy
}

\author{
Carsten Cleff, Petra Groß, Sven Dobner, and Carsten Fallnich \\ Institute of Applied Physics, Westfälische Wilhelms-Universität Münster, Corrensstraße 2, 48149 Münster, Germany \\ Herman L. Offerhaus and Jennifer L. Herek \\ Optical Sciences Group, MESA+ Research Institute for Nanotechnology, University of Twente, P.O. Box 217, \\ Enschede NL-7500 AE, The Netherlands \\ Kai Kruse, Willem P. Beeker, Chris J. Lee, and Klaus-Jochen Boller \\ Laser Physics \& Nonlinear Optics Group, MESA+ Research Institute for Nanotechnology, University of Twente, \\ P.O. Box 217, Enschede NL-7500 AE, The Netherlands \\ c.cleff@wwu.de
}

\begin{abstract}
Suppression of CARS signal generation is demonstrated by equalization of the ground and Raman states via a control state in a theoretical investigation. Using donut-shaped control light fields for population transfer results in sub-diffraction-limited spatial resolution CARS microscopy.
\end{abstract}

(C) 2012 Optical Society of America

OCIS codes: $100.6640,180.4315,300.6230$.

\section{Introduction}

Coherent anti-Stokes Raman scattering (CARS) microscopy is a powerful technique with intrinsic chemical selectivity, which makes CARS suited for in situ imaging of, e.g., biological samples and drug dissolution processes, but also for the investigation of nanostructures. As a nonlinear microscopy technique, CARS enables three-dimensional imaging with high spatial resolution [1], it is, however, still limited by diffraction and thus confined to imaging above the diffraction limit.

Here we present a theoretical investigation of sub-diffraction-limited spatial resolution in CARS microscopy by stimulated emission pumping from the ground state to the Raman state via a vibrational or electronic control state prior to the CARS signal generation. The population equalization of the ground and Raman state results in suppression of CARS signal generation. This scheme does not require any restrictive molecular properties, rendering this approach a generally applicable scheme.

\section{Theoretical framework}

Our theoretical investigation is based on a density matrix formalism approach to describe a molecular four-level model system. The CARS signal generation was calculated by evaluating the temporal evolution of the coherences between the four states. A more detailed description of the density matrix approach is given in [2]. The modeled four-level system consists of the ground state, an electronic state and two low energy states to which we refer as vibrational and Raman (for the state involved in the CARS signal generation) state. Either the electronic or the vibrational state is used as control state for the population transfer from the ground state to the Raman state. Transitions from the ground state to the vibrational and electronic state as well as transitions from the electronic state to the Raman state are dipole allowed while all other transitions are dipole forbidden. In contrast to our previous work [2], here no specific molecular properties are needed to achieve a suppression of the CARS signal.

In order to equalize the ground and Raman state, a control light field pulse pair is applied simultaneously. By the first control light field population is transferred from the ground state to the control state and by the second one the transition from the control state to the Raman state is stimulated - which is also known as stimulated emission pumping. The pulse duration of the control light fields is chosen to be longer than the coherence lifetime of the control state to 

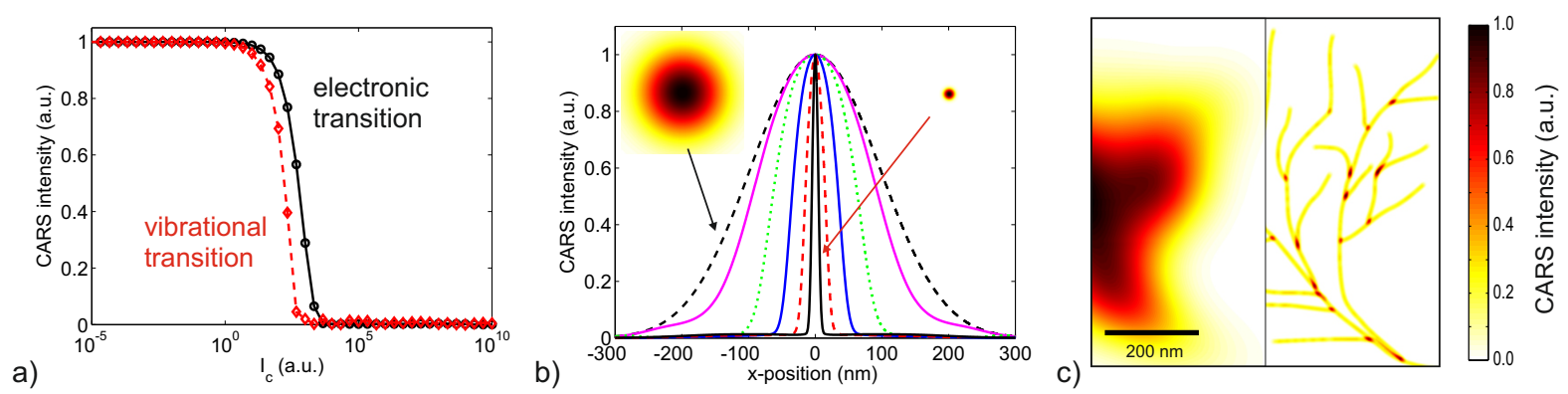

Fig. 1. a) CARS intensity as a function of the control light field intensity. b) CARS excitation profiles with donut-shaped control light fields applied. Increasing the control light field intensities results in a successive narrowing below the diffraction limit. Two-dimensional illustrations of two profiles are shown in the insets. c) CARS image without (left) and with (right) applied control light field pair of the same image section. The right image clearly resolves sub-diffraction-limited structures of the test sample.

avoid coherent effects such as Rabi oscillations [3]. In this way an equal population in the ground and Raman state is achieved prior to the CARS signal generation. As a result, during the CARS excitation the population transfer from the ground state to the Raman state and a build-up of vibrational coherence between this states is reduced, leading to a suppression of CARS signal generation.

\section{Results and Discussion}

By applying a pulsed control light field pair we observe an equalization between the population of the ground and Raman states as well as a strong population of the control state. Fig. 1 a shows the resulting CARS signal generation as a function of the intensity of the control light field pair for the case that the vibrational state acts as control state (red dashed curve) and that the electronic state acts as control state (black solid curve). In both cases the CARS signal is significantly suppressed, when a certain intensity of the control light field pair is reached, and saturates at a CARS signal suppression of more than $99.8 \%$. The suppression of the CARS signal at lower intensities of the control light field pair in the case of the vibrational state acting as control state results from different coherence decay rates of the transitions to the vibrational and electronic state, respectively.

We calculate CARS excitation profiles for different donut peak intensities using the electronic state as control state and assuming a donut-shaped spatial profile for the control light field pair. With increasing control light field intensity the excitation profile narrowed monotonously below the diffraction limit (see Fig. $1 \mathrm{~b}$ ). CARS images resulting from these excitation profiles were simulated based on a computer-generated test object resembling a dendritic line structure with spatial features below the diffraction limit of $225 \mathrm{~nm}$. Fig. $1 \mathrm{c}$ shows on the left side a CARS image of the test image calculated with a diffraction-limited excitation profile (Fig. 1 b black dashed curve, FWHM of $225 \mathrm{~nm}$ ) and on the right side a CARS image of the same test image calculated with a significantly narrowed CARS excitation profile (Fig. $1 \mathrm{~b}$ black solid curve, FWHM of $15 \mathrm{~nm}$ ). While in the left image no features of the line structure are visible, those are clearly revealed in the right image, demonstrating sub-diffraction-limited resolution.

In conclusion, we have presented a way of achieving sub-diffraction-limited resolution in CARS microscopy based on stimulated emission pumping using a pulsed control light field pair. Either an electronic or vibrational state can be used as control state allowing some flexibility for the control light field frequencies. This way a frequency regime can be chosen where the sample damage threshold is high, allowing to optimize the spatial resolution that can be achieved.

\section{References}

1. A. Zumbusch, G. R. Holtom, and X. S. Xie, Phys. Rev. Lett. 108, 827 (2004).

2. W. P. Beeker, P. Groß, C. J. Lee, C. Cleff, H. L. Offerhaus, C. Fallnich, J. L. Herek, and K.-J. Boller, Opt. Express 17, 22632 (2009).

3. W. P. Beeker, C. J. Lee, K.-J. Boller, P. Groß, C. Cleff, C. Fallnich, H. L. Offerhaus, and J. L. Herek, Phys. Rev. A 81, 012507 (2010). 